\title{
IMMEDIATE VERSUS DELAYED LOADING IN ALL ON FOUR IMPLANT SUPPORTED HYBRID PROSTHESIS
}

\author{
Amr Salah El-din Gomaa* and Ahmed Mohamed Osama **
}

\begin{abstract}
Objectives: the aim of the study is to evaluate radiographically the crestal bone height change around all on four implant supported hybrid prosthesis

Material \& Methods: Fourteen patients were selected from Ain shams Outpatient. clinics having completetly edentulous maxilla opposed by full dentulous mandible and they were properly diagnosed for implant placement. Implant sites were planned virtually and a 3D printed surgical guide was made. The implant had been installed in proper position and angulations using the surgical guide. Patients were randomly divided into 2 groups, Group I; received a hybrid prosthesis on delayed loaded four implants, Group II; received a hybrid prosthesis on immediately loaded four implants. Postoperative CBCT was performed at time of pickup (base line), 6 and 12 months

Results: Although the data obtained revealed that group I showed lower level of crestal bone height change compared to group II, statistical analysis of the data revealed insignificant difference during the one year follow up period.

Conclusion: in all on four implants delayed loading hybrid prosthesis shows no significant difference regarding bone resorption around implants than in all on four implants immediately loaded hybrid prosthesis
\end{abstract}

KEYWORDS: immediate loading, delayed loading, hybrid prosthesis, all on four implants

\section{INTRODUCTION}

Patients prefer an implant supported fixed prosthesis rehabilitation for maxillary edentulous arch as they are annoyed by the coverage of the palatal vault with a conventional denture that prevents temperature and taste sensation and increase gagging reflex and swallowing problems occur in conventional removable prosthesis but this prosthesis requires sufficient number of implant for a successful prosthesis .(1)

On the other way implant-assisted overdenture. are supported, retained, and stabilized by both

* Lecturer of Removable Prosthodontics, Faculty of Dentistry, Fayoum University.

** Associate professor of Removable Prosthodontics, Faculty of Dentistry- Ain shams University. 
implants and mucosa; therefore they generally require fewer implants than fixed implant prosthesis. In the maxilla, four axially inclined endosseous implants, are considered the minimum number needed for overdenture treatment with palatal coverage and proper extension of the overdenture. ${ }^{(2)}$

However, dental implants are traditionally placed in the vertical position. But. with minimum bone volume, poor bone quality and the need for bonegrafting and sinus lifting procedures before implant placement create some challenging conditions. The use of distal tilting of implants may be advantageous. Tilting preserves relevant anatomical structures and allows for placement of longer implants with good cortical anchorage in optimal positions for prosthetic support inclined implant can reduce the force acting over the implants, also increases the inter-implant space, reduces cantilever length in jaws. ${ }^{(3, \ldots . . . .6)}$

That is why angulated implants placement for rehabilitation of patients with severely resorbed jaws and named this concept as "All on four" in which two vertical implants are placed in anterior region and two implants in posterior region are placed at an angulation of 35-40 degrees. Since then, several clinicians and researchers have reported use of angulated implants with varying degrees of success over a period of time.$^{(6 \ldots .11)}$

The distally tilted implant increase the Stability in minimum bone volume as longer implants can be used which increase bone-to-implant contact and decrease the need for vertical bone augmentation, and bone grafting. All on 4 concept is effective and safe alternative to maxillary sinus floor augmentation and sinus lifting procedures, which are invasive with unpredictable outcome. ${ }^{(12)}$

Moreover; in all on four concept the Implants are well-spaced, easier to clean, provide immediate function and esthetics Final restoration can be fixed or removable and decreases in cost due to less number of implants are used, which solve many problems but it is highly. Technique sensitive , need very skillful operator to perform it as Free Hand arbitrary surgical Placement of implant is not always possible even slight change in angulation may cause a problem to patient and operator. Implant placement is completely prosthetically driven which requires accurate pre-surgical preparation such as standardized all on four surgical guide with conventional flap technique or Computer guided surgical stent flapless technique to place implants in the desired angulations. ${ }^{(13 \& 14)}$

Computer generated surgical guide have been evolved to overcome the limitations associated with conventional radiographic surgical template Computer guided surgery provides a link between the treatment plan and the actual surgery by transferring the simulated plan accurately to surgical site This surgical guide is made using stereolithography process and is custom manufactured for each patient. ${ }^{(15)}$

The surgical guides fabricated by Stereolithography are preprogrammed with Individual depth, angulations, mesio-distal and labiolingual positioning of the implant. ${ }^{(16)}$

Fabrication of stereolithographic surgical guide requires patient's computed tomography (CT) image. In CT, multi planar reformatting allows one to reformat a volumetric dataset in sagittal, axial, and coronal cuts and also helps in building multiple cross-sectional and panoramic views. ${ }^{(17)}$

Special planned Software has been adapted to allow practitioners to virtually view the implant site and plan location, angle, depth, and diameter of virtual implants, which are superimposed on the 3D data. Following backward planning, the diagnostic wax up has to be visualized through CT scan with radiographic templates in place. ${ }^{(18 \& 19)}$

\section{MATERIAL \& METHODS}

Fourteen patients that require dental implant placement had Participated in this study. Their age ranged from 40 to 70 years. The participants were recruited from Outpatient clinics, Faculty of Dentistry, Ain shams University. 
Patient recruited in the study fulfilled the Following inclusion criteria: all patients had edentulous maxilla for more than 1 year opposed by dentulous mandible; sufficient bone in height and width; sufficient attached mucosa around implant; sufficient mouth opening greater than $50 \mathrm{~mm}$ to accommodate space to the surgical guide; in a good general health while exclusion. Criteria for this study serve all the medical conditions that maks the implant insertion unfavorable; as the need for bone grafting and / or sinus lift in the planned area; and patients unlikely to be able to comply with study procedures.

\section{Construction of single denture:}

Upper and lower primary impressions were made. A provisional centric relation was recorded to mount maxillary and mandibular casts for setting up of the upper artificial teeth Opposing lower natural teeth interfering with artificial teeth were adjusted on the cast and accordingly modified in the patient's mouth with a clear acrylic template which was fabricated on the modified lower stone cast. The fitting surface of the template was coated with pressure indicating paste and seated on lower mandibular natural teeth, Interferences were detected through the template and removed by reshaping occlusal anatomy.

In the pre-constructed special trays for the upper arch secondary impression was made. Border tracing was done using green stick compound * and the secondary impressions were made using zinc oxide/Eugenol impression ${ }^{* *}$ material Secondary impression was poured in dental stone ${ }^{* * *}$ to obtain the master cast.

- Upper occlusion block was constructed. Centric occluding relation was recorded following the wax wafer technique to mount the maxillary and mandibular cast were mount on mean value articulator $^{* * * *}$. Modified cross-linked acrylic teeth $^{* * * * *}$ were arranged following the group functioned concept of occlusion anteriorly.

- The trial denture was tried in the patient's mouth to ensure proper facial contour, aesthetic, even contact between all the posterior teeth and harmony between centric occlusion and centric relation at the predetermined vertical dimension of occlusion. The waxed up denture was flasked and processed into heat-cured acrylic resin.

- Upper single complete denture was fabricated with proper flange extentension and delivered to the patient.

\section{Surgical and Prosthetic planning:}

\section{Virtual implant planning and sterolithographic surgical guide fabrication fig(1):}

\section{A-Dual scan protocol}

- Virtual planning began with dual scan protocol by modifying patient's upper complete single denture into radiographic guide by adding seven spherical radiographic fiducially markers ${ }^{* * * * * * *}$. The two scans were superimposed onto each other and the CBCT raw data was converted into $3 \mathrm{D}$ information by in 2guide cyber med software ${ }^{* * * * * * * *}$ where the final file contained reformatted images in 3D bone model, 3D radiological dataset and 3D radiographic modified denture guide model .

- Four conventional interactive* two piece implants screw type with $3.7 \mathrm{~mm}$ in diameter and $10 \mathrm{~mm}$ in length were selected to follow

\footnotetext{
* Perfectin., S.A.I.C., HUBAC, .BUENESAIRES, .Argentina

** Cavex impression. paste, Cavex. Hollad by. P.O. Box 852, 2003 RW.

*** lab stone, Miles dental. product, Miles, INC, South bend,USA.

****** Artec. proarticulator, Milano, Italy

***** Vita-pan acrylic. teeth, Vita Bad Sackingen- Germany .

****** Faber. castell , Blu Tack Reusable Removable Adhesive Glue ,Germany

$* * * * * * * 2$ guide cyber med .software, Seoul ,Korea.
} 
all on 4 concept, 2 anterior straight implants with 17 degree multi unit abutments and 2 posterior mesially tilted implants with 30 degree angulated multi unit abutments .

\section{B- Sterolithographic surgical guide fabrication}

-Stereolithographic apparatus Envision tec DDDP was use which has vat, Polymerization process of photo polymerized resin occured in layers.

\section{Surgical protocol}

- Surgery was performed under local infiltration anesthesia technique with articaine chlorohydrate with adrenaline 1: 100,000** away from areas where guide was based .

- The mucosal supported surgical guide was adapted by occlusion with the opposite arch. Fixation of the guide was done by using the manufacture provided surgical guide surgical kit***

- The drilling sequence for implant placement began with a tissue punch though each metal sleeve of the surgical guide and then sequential drilling was done for each implant through the surgical guide After the final implants have been positioned, the final rotational position and depth are accomplished by a torque controller with a torque limit $50 \mathrm{Ncm}$ and intail stability of the implants were musered using Osstell ${ }^{* * * *}$. Fig $(1,2 \& 3)$

\section{C) Grouping of the patients:}

Patients were randomly divided into two equal groups

Group I: received hybrid prosthesis on delayed loaded implants.

Group II: received a hybrid prosthesis on immediately loaded 4 implants

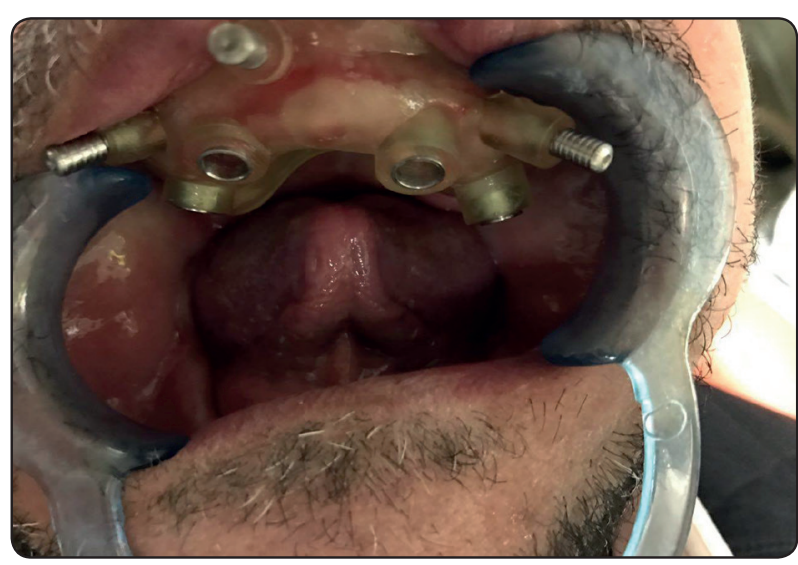

Fig. (1) Surgical stent fixed in place ready for drilling

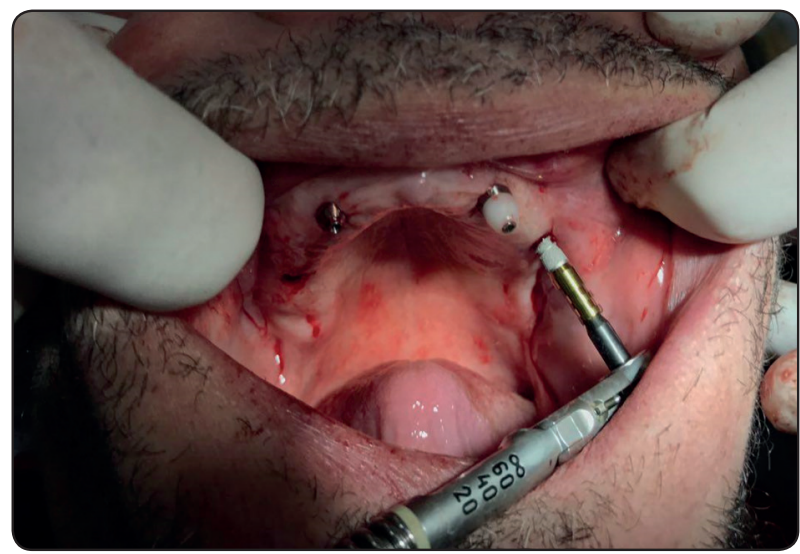

Fig. (2) Implant insertion

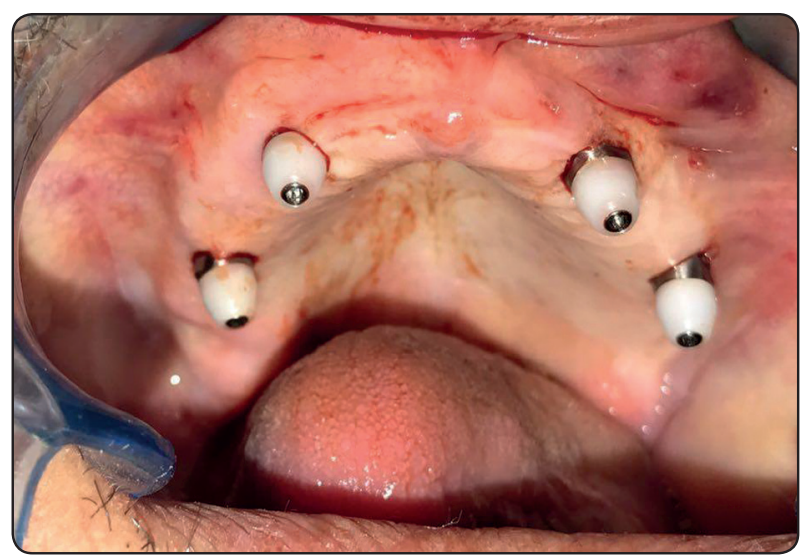

Fig. (3) All four implants are in place

\footnotetext{
* Implant direct. conical connection ,USA

** Septodont ,.Saint -Maur,France.

*** In2 guide universial .kit cyber med ,Seoul ,Korea

**** Osstell ISQ. device, Gothenburg, Sweden.
} 
At the time of loading a multi unit abutments were screwed to the posterior implants and anterior abutments were screwed to the anterior implants with torque $25 \mathrm{~N}$. Parallel platform between abutments were seen to which the denture could be attached.

\section{Transformation of upper complete denture into interim hybrid fixed processes}

\section{-Placement of titanium sleeves}

- Index position of the multi unit abutments were done using in fitting surface of already presented upper complete denture.

- Four holes were made in denture for titanium sleeves located by bite material* holes were large enough and rechecked intraoral for proper seating and passive fit.

\section{D) Pick up of titanium sleeves:}

- Cold curing acrylic resin * was used to pick up the titanium sleeves in the upper denture Cold curing acrylic resin was injected around the sleeves after proper blocking of under cuts using small pieces of rubber sheets around titanium sleeves and with the holes created in the denture. The denture was seated, excess resin on the top access of the sleeves was removed and the patient was asked to bite incentric occlusion till the resin was totally set .

- Cotton pieces positioned on the top of the sleeves was removed with explorer probe and with a unigrip screw driver the prosthesis was unscrewed, and then complete upper denture is converted to fixed hybrid prosthesis, the palatal portion, borders, and flanges were trimmed. A sharp angles or edges were removed too.

-The tissue side of the acrylic provisional prosthesis was made convex and polished. The acrylic provisional prosthesis was then screwed back to the multi unit abutments in the mouth occlusion was rechecked with articulator paper to be adjusted.

-A CBCT was made immediately and used to be superimposed with the preoperative $\mathrm{CBCT}$ to ensure accuracy of the guide and correct positioning of the implants.

-Patients were recalled every two weeks for a period of three months so the titanium sleeves were unscrewed and monitoring condition of soft tissue and hygiene condition was monitored.

-Patients were recalled every Six months for follow up radiographic evaluation.

\section{E) Follow up:}

-Cone beam radiographs CBCT was taken to evaluate the marginal bone height change around the four implants in each case on both groups immediately after loading, six months and one year.

-The CBCT used is a Planmeca classic, with 66-90kV, 1-14 mA., resolution 100-150 voxels Eighteen sec examination time was needed. Dose. per scan was $100 \mathrm{mSv}$.

-Measurements were taken at mid buccal, mid lingual, distal and mesial to evaluate the bone loss around the implants and then all data were collected, tabulated and statistically analyzed to evaluate the effect of two treatment modalities on the crestal bone level surrounding the implants.

\section{RESULTS}

The results of this study are shown in tables (1) and figures (4). Numerical data were explored for normality by checking the data distribution, calculating the mean and median values, evaluating histograms and normality curves and using olmogorov-Smirnov and Shapiro-Wilk tests.

\footnotetext{
*3M ESPE imprint 4 bite. VPS bite registration .material, Germany

**Chair side hard denture. relining material Promedica. dental material Gmbh ,Germany.
} 
Data were assumed non parametric, Data were presented by mean, standard deviation (SD). Wilcox on signed rank test was used for comparison between follow up periods. The significance level was set at $P \leq 0.05$. Statistical analysis was performed. with IBM ${ }^{\circledR}$ SPSS $^{\circledR}$ Statistics Version 20 for Windows. Mann Whitney test was used for comparison between two groups

Group I : received hybrid prosthesis on delayed loaded implants.

Group II: received a hybrid prosthesis on immediately loaded 4 implants

Crestal bone height change in group I and group II during one year follow up period.

TABLE (1): The mean and standard deviation values of crestal bone height change around the implants for Group I and Group II during follow up periods.

\begin{tabular}{|c|c|c|c|c|c|}
\hline & \multicolumn{2}{|c|}{ Group I } & \multicolumn{2}{|c|}{ Group II } & \multirow{2}{*}{$\begin{array}{c}\mathrm{P} \\
\text { value }\end{array}$} \\
\hline & Mean & $\begin{array}{c}\text { Std. } \\
\text { deviation }\end{array}$ & Mean & $\begin{array}{c}\text { Std. } \\
\text { deviation }\end{array}$ & \\
\hline $\begin{array}{l}\text { Baseline- } \\
6 \text { months }\end{array}$ & 0.44 & 0.429 & 0.56 & 0.37 & 0.601 \\
\hline 6-12months & 0.35 & 0.25 & 0.49 & 0.15 & 0.244 \\
\hline $\begin{array}{l}\text { Baseline } \\
-12 \text { months }\end{array}$ & 0.8 & 0.404 & 1.05 & 0.33 & 0.226 \\
\hline
\end{tabular}

- Six months after loading the mean value amount of crestal bone height change was 0.44 and 0.56 for group I and group II respectively.

- During interval 6-12 month follow up the measured amount of crestal bone height change was 0.35 and 0.59 for group I and group II respectively.

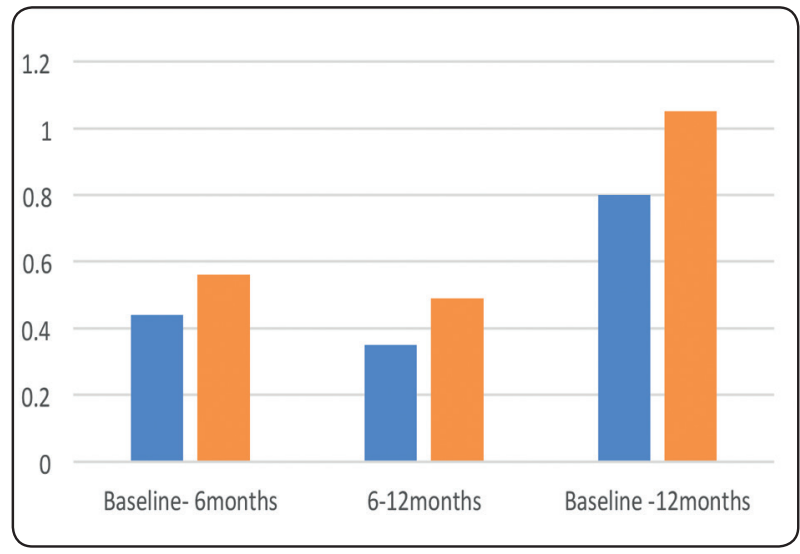

Fig. (4): Mean values of bone height.change of two studied groups at different follow up periods

- At the end of the one year follow up period the amount of crestal bone height change was 0.8 and 1.05 for group I and group II respectively.

- Although the data obtained from the table (1) and figure (4) revealed that group I showed lower level of crestal bone height change compared to group II, statistical analysis of the data revealed insignificant difference during the one year follow up period .

\section{DISCUSSION}

The conversion of the patient's satisfactory existing denture to a screw-retained implant fixed prosthesis was described to splint implants together using acrylic resin denture base and enables the clinician to deliver the prosthesis in a few hours. ${ }^{(20)}$

Also, the patient can wear and evaluate the appearance, speech, and comfort of the prosthesis and ask for changes in the final prosthesis to be placed after the healing period. The conversion of the patient's denture can be performed chairside. Acrylic resin being considered a semi-rigid material, the prostheses without the cast metal bar still were rigid enough to not have a negative effect on the implant stability. ${ }^{(21)}$ 
Cone beam radiograph was used to asses alveolar bone height and its loss around implants Cone beam was used as it provide accurate $3 \mathrm{~d}$ image, with minimal dose of radiation compared to conventional. CT system, inexpensive, and limited scan time. ${ }^{(22)}$

Success of dental implant treatment mainly depends on the sustainable long-term health of soft and hard peri implant tissues Assessment of mobility, pain, infection, inflammation and marginal alveolar bone loss are all considered as useful implant success criteria. Specific attention has been directed towards post-operative radiographic assessment of marginal alveolar bone loss around implants by serial radiographs Vertical marginal bone loss at the peri-implant surfaces should not exceed $1-2 \mathrm{~mm}$ during the first year of function and $0.2 \mathrm{~mm}$ thereafter. ${ }^{(23 \& 24)}$

The Improvement of oesseointegration treatment with dental implants is only considered a complete success when implants are oesseointegrated with absence of pain, inflammation, lack of mobility, absence of continuous per apical radiolucency and return of esthetics and functions.$^{(25)}$

Bone formation at the implant Bone interface is a complex physiological process regulated by systemic hormones and local factors produced by the skeletal cells. It involves sequential events such as cellular attachments, proliferations, differentiations and the deposition of bone Matrix, moreover criteria for successful implant therapy include a median marginal bone loss of $0.5 \mathrm{~mm}$ during healing, followed by an annual rate of vertical bone loss of less than $0.2 \mathrm{~mm}$ a year, while the longevity of dental implants is highly dependent on integration between implant components and oral tissues, including hard and soft tissues. The implants show 0.9 to $1.6 \mathrm{~mm}$ marginal bone loss from the first thread by the end of the first year in function, while only 0.05 to 0.13 $\mathrm{mm}$ bone loss occurred after the first year. ${ }^{(26,27)}$

\section{REFERENCE}

1- Marco. Tallarico, Lecturer; Silvio. Mario Meloni, Luigi Canullo, Martina Caneva, Giovanni. Polizzi: five-year results of a randomized. controlled trial comparing patients rehabilitated. with immediately loaded maxillary crossarch fixed dental prosthesis supported by four or six implants placed using guided surgery. Clinical Implant Dentistry 966 Clinical Implant dentistry and related research, vol 18, number 5,2016 .

2- Feine, J.S., Carlsson, G.E.: Implant Overdentures: The Standard of Care for Edentulous Patients. Quintessence: Carol Stream, IL. 2003.

3- Charles A. Babbush, Gary T. Kutsko. „John Brokloff : The all on four immediate function treatment concept with Nobel active implants : a retrospective study. J of Oral Implantology, 2011.

4- Vijay Ebenezer and Balakrishnan: Angulated implants: a literature review. International $\mathrm{J}$ of Recent Scientific Research ,Vol. 8, Issue, 9, pp. 20154-20158, September, 2017.

5- Bilhan H.: An alternative method to treat a case with severe maxillary atrophy by the use of angled implants instead of complicated augmentation procedures: a case report. Journal of Oral Implantology,34 (1):47-51. Pub Med, 2008.

6- Lim TJ, Csillag A, Irinakis T, Nokiani A, Wiebe CB. : Intentional Angulation of an Implant to Avoid a Pneumatized Maxillary Sinus: A Case Report. J Can Dent Assoc., 70(3):164-68. Pub Med, 2004.

7- Taschieri S, Fabbro MD, Tsesis I, Corbella S.: Maxillary Sinus in relation to Modern Oral and Maxillofacial Surgery. International Journal of Dentistry,2012.

8- John Beumer III : Fundamentals of implant dentistry, Quintessence Publishing CO, Inc 2015.

9- Del Fabbro M, Bellini CM, Romeo D, Francetti L.:Tilted implants for the rehabilitation of edentulous jaws: a systematic review Clin. Implant Dent Relat Res,May 2010.

10- Malo’ P, Rangert B., Nobre M. :All-on-Four immediatefunction. concept with Branemark system. implants for completely edentulous. mandibles: a retrospective clinical. study. Clin Implant Dent Relat Res,2003.

11- Malo' P, Rangert B., Nobre M.: All on 4 immediate function. concept with Branemark system. implants for completely edentulous maxillae: a 1-year retrospective. clinical study. Clin Implant Dent. Relat Res,2005. 
12- B. Anandh, B.Lokesh, Vijay Ebenezer, S. Jimson and J. Parthiban: All on four - the basics. Bio medical \& pharmacology J vol. 8, p 609-612, Oct. 2015.

13- Malo P, Lopez I., Nobre M. :The All on Four. concept. In: Babbush C, Hahn J, Krauser. J, eds. Dental Implants: The Art and Science. 2nd ed. St Louis, Mo: Saunders Elsevier; 2010.

14- Calandriello. R, Tomatis M.:Simplified treatment of the atrophic posterior maxilla via immediate/early function and tilted implants: a prospective 1-year clinical study. Clin Implant Dent Relat Res.,2005.

15- Weinstein R., Agliardi E, Fabbro MD., Romeo D, Francetti L.: Immediate rehabilitation of the extremely. atrophic mandible with fixed. full-prosthesis supported by four implants. Clin Implant. Dent Relat Res.,2010.

16- Vijay Ebenezer. and Balakrishnan: Angulated. implants: a literature review. International $\mathrm{J}$ of Recent. Scientific Research, Vol. 8, Issue, 9, pp. 20154-20158, September, 2017.

17- B. Anandh., B.Lokesh, Vijay Ebenezer., S.Jimson and J.Parthiban.: All on four - the basics. Bio medical. \& pharmacology J vol. 8,p 609-612, Oct. 2015.

18- Widmann G., Bale RJ. :Accuracy. in computer-aided implant surgery: A review. Int J Oral Maxillofac. Implants, 21:305-13,2006.

19- Lal K, White GS., Morea DN and Wright. RF.: Use of stereolithographic templates for. surgical and prosthodontic implant. planning and placement. Part I. The concept J Prosthodont. , 15:51-8, 2006.

20- Manikandan Ramasamy., Ramesh Rajaand. Subramonian, Karthik : Implant surgical. guides: From the past to. the present. J of Pharmacy \& Bio allied Sciences. ;Jun 2013.
21- Eloana. Thome,Hyung Joo. Lee, Ivete Aparecida. de Mattias, Sartori Roseli Latenek. Trevisan ,Jaques Luiz. and Rodrigo Tiossi: A randomized controlled. trial comparing interim acrylic prostheses. with and without cast metal base for immediate loading. of dental implants in the edentulous mandible. Clin. Oral Impl. Res. 26, 2015 .

22- Scarfe, W.C., Farman, A.G., and Sukovic, P.: Clinical applications of cone-beam computed. tomography in dental. practice. J Can Dent Assoc, 72(1): p. 75-80,2006.

23- K Kamburo־glu., S Murat, C Kılıç., S Y“* uksel, H Avsever., A Farman and W C Scarfe: Accuracy of CBCT images. in the assessment of buccal marginal alveolar peri-implant. defects: effect of field of view; Dentomaxillofacial Radiology, 2014.

24- Kamburo glu K., Gulsahi A, Genç Y, Paksoy CS.: A comparison of peripheral marginal bone loss at dental implants. measured with conventional intraoral film and digitized radiographs. J Oral Implantol, 38: 211-19, 2012.

25- patrica M .Freitas. and Alyne Simoes : Laser In Dentistry. Guide for Clinical Practice ; published. by John Wiley and Sons,Inc 2015.

26- Hans Joachim. Nickenig, Manfred Wichmann., Karl Andreas Schlegel ,Emeka Nkenke. and Stephan. Eitner: radiographic evaluation of marginal bone levels adjacent. to parallel-screw cylinder machined-neck implants and rough-surfaced microthreaded. implants using digitized panoramic radiographs; Clin. Oral Impl. Res. 20, 2009.

27- Mohammed. A. Alshehri: The maintenance of crestal bone. around dental implants. International. magazine of oral implantology vol 12, issue 2,2011. 